\title{
Algumas perspectivas para análise da gestão da saúde no estado de São Paulo (Brasil)
}

\author{
Some perspectives for the analysis of health management \\ in São Paulo State (Brazil)
}

Regina Célia Ermel ${ }^{1}$

Fernanda Martins Bigio ${ }^{1}$

Aline Francine Raphael Evangelista ${ }^{1}$

Maria Fernanda Pereira Gomes ${ }^{1}$

Lislaine Aparecida Fracolli ${ }^{2}$

${ }^{1}$ Universidade de Marília. Av. Higyno Muzzi Filho 1.001. 17525-902 Marília SP.rcermel@terra.com.br ${ }^{2}$ Departamento de Saúde Coletiva, Escola de Enfermagem, Universidade de São Paulo.

\begin{abstract}
The current study aims to reflect the changes made in Secretaria do Estado da Saúde de São Paulo (SES) (São Paulo's Health State Secretariat, Brazil) related to the theoretical management proposals. A brief bibliography research was done, in Lilacs and Scielo databases. Twenty two bibliography sources from the period of 1993 to 2007 were selected. The results have shown that the main trends in the management of public administration are represented by the separation between the political and administrative functions, distinction between state exclusive activities from the ones that can be accomplished by other workers, emphasis of procedure control to product control, clear result attribution, definition of rendering account forms, governability and governing. It has been concluded that the redefinition and direction of several functions of the SES' management allowed the continuity of the decentralization processes so that health actions coverage is enlarged and it makes possible the attention inequalities decrease and promotes the equality in the health processes through an articulated management between states and cities.
\end{abstract}

Key words Health manager, Decentralization, Equity
Resumo O presente estudo visa refletir sobre as reformas efetuadas na Secretaria de Estado da Saúde de São Paulo (SES) ante as propostas teóricas de gestão. Realizou-se uma breve pesquisa bibliográfica, na base de dados Lilacs e Scielo. Selecionaram-se 22 fontes bibliográficas pertencentes ao período de 1993 a 2007. Os resultados mostraram que as principais tendências no que concerne à administração pública gerencial são representadas pela separação entre funções políticas e administrativas, distinção entre atividades exclusivas de estado das que podem ser realizadas por outros agentes, ênfase do controle dos procedimentos para controle dos produtos, atribuição clara de resultados, definição de formas de prestação de contas, governabilidade e governança. Concluiuse que a redefinição e o direcionamento das diversas funções de gestão da SES possibilitaram a continuidade dos processos de descentralização com o intuito de que a cobertura das ações de saúde fosse ampliada diminuindo as desigualdades de atenção e promovendo a equidade nos processos de saúde através de uma gestão articulada entre o estado e os municípios.

Palavras-chave Gestor de saúde, Descentralização, Equidade 


\section{Introdução}

O documento das diretrizes do Pacto pela Saúde em 2006 - Consolidação do Sistema Único de Saúde (SUS) -, publicado na Portaria/GM no 399, de 22 de fevereiro de 2006, contempla o pacto firmado entre os gestores do SUS em suas três dimensões: pela Vida, em Defesa do SUS e de Gestão ${ }^{1}$. Esse pacto apresenta mudanças significativas para a execução do SUS, dentre as quais se ressaltam: a substituição do atual processo de habilitação pela adesão solidária aos termos de compromisso de gestão; a regionalização solidária e cooperativa como eixo estruturante do processo de descentralização; a integração das várias formas de repasse dos recursos federais; e a unificação dos vários pactos existentes.

Foi o resultado de um intenso trabalho de discussão de cerca de dois anos, envolvendo os técnicos e a direção das diversas áreas do Ministério da Saúde, do Conselho Nacional de Secretários Municipais de Saúde (Conasems) e do Conselho Nacional de Secretários de Saúde (Conass) e foi aprovado na reunião da Comissão Intergestores Tripartite de 26 de janeiro de 2006 e na reunião de 9 de fevereiro de 2006 pelo Conselho Nacional de Saúde.

O Pacto pela Vida é o compromisso entre os gestores de prioridades que apresentam impacto sobre a situação de saúde da população brasileira. A definição de prioridades deve ser estabelecida por meio de metas nacionais, estaduais, regionais ou municipais. São seis as prioridades pactuadas: saúde do idoso; controle do câncer de colo do útero e de mama; redução da mortalidade infantil e materna; fortalecimento da capacidade de resposta às doenças emergentes e endemias, com ênfase na dengue, hanseníase, tuberculose, malária e influenza; promoção da saúde; e fortalecimento da Atenção Básica.

O Pacto em Defesa do SUS estabelece como diretrizes: os compromissos entre os gestores do SUS com a consolidação da Reforma Sanitária Brasileira e o desenvolvimento e articulações de ações, no seu âmbito de competência e em conjunto com os demais gestores.

O Pacto pela Gestão estabelece como diretrizes os aspectos de descentralização; regionalização; financiamento; planejamento; programação pactuada e integrada (PPI); regulação; participação e controle social; gestão do trabalho e educação na saúde.

O processo de descentralização estabelece que cabe ao Ministério da Saúde a proposição de políticas, a participação no cofinanciamento, co- operação técnica, avaliação, regulação, controle e fiscalização, além da mediação de conflitos. Este sistema de descentralização estabelece ainda que as deliberações das Comissões Intergestores Bipartite e Tripartite devem ser por consenso, sendo que as Comissões Intergestores Bipartite são instâncias de pactuação e deliberação para a realização dos pactos intraestaduais e definição de modelos organizacionais, a partir de diretrizes e normas pactuadas na Comissão Intergestores Tripartite, e a Comissão Intergestores Tripartite e o Ministério da Saúde promoverão e apoiarão o processo de qualificação permanente para as Comissões Intergestores Bipartite.

A regionalização é uma diretriz do SUS, um eixo estruturante do Pacto de Gestão, e deve orientar a descentralização das ações e dos serviços de saúde, os processos de negociação e pactuação entre os gestores.

Com relação ao financiamento do SUS, estabeleceu-se que este é de responsabilidade das três esferas de gestão (União, estados e municípios), sendo que o repasse, fundo a fundo, ficou definido como modalidade preferencial de transferência de recursos entre os gestores e que o financiamento do custeio com recursos federais será constituído, organizado e transferido em blocos de recursos. Os blocos de financiamento para o custeio são: Atenção Básica; Atenção de Média e Alta Complexidades; Vigilância em Saúde; Assistência Farmacêutica e Gestão do SUS.

O processo de planejamento no âmbito do SUS deve ser desenvolvido de forma articulada, integrada e solidária entre as três esferas de gestão. Este sistema de planejamento pressupõe que cada esfera de gestão realize o seu, articulando-se de forma a fortalecer e consolidar os objetivos e diretrizes do SUS, contemplando as peculiaridades, necessidades e realidades de saúde locorregionais ${ }^{1}$.

O sistema de planejamento buscará, de forma tripartite, a pactuação de bases funcionais do planejamento, monitoramento e avaliação do SUS, bem como promoverá a participação social e a integração intra e intersetorial, considerando os determinantes e condicionantes de saúde. No cumprimento da responsabilidade de coordenar o processo de planejamento, levar-se-ão em conta as diversidades existentes nas três esferas de governo, de modo a contribuir para a consolidação do SUS e para a resolubilidade e qualidade, tanto da sua gestão quanto das ações e dos serviços prestados à população brasileira ${ }^{1}$.

A regulação da atenção à saúde tem como objetivo a produção de todas as ações diretas e 
finais da atenção à saúde, dirigida aos prestadores de serviços de saúde, públicos e privados. As ações da regulação da atenção à saúde compreendem a contratação, a regulação do acesso à assistência ou regulação assistencial, o controle assistencial, a avaliação da atenção à saúde, a auditoria assistencial e as regulamentações da vigilância epidemiológica e sanitária.

A participação e o controle social são ações que devem ser desenvolvidas para fortalecer o processo de participação social dentro deste pacto visando apoiar os Conselhos de Saúde, processo de formação de conselheiros, estimular a participação e avaliação dos cidadãos, apoiar o processo de educação popular na saúde, apoiar a implementação de ouvidoria nos municípios e estados e apoiar o processo de mobilização social e institucional em defesa do SUS.

Na Gestão do Trabalho, a política de recursos humanos para o SUS é um eixo estruturante e deve buscar a valorização do trabalho e dos trabalhadores da saúde, o tratamento dos conflitos, a humanização das relações de trabalho, e como diretriz, o Pacto de Gestão preconiza a educação permanente como política de formação e desenvolvimento dos trabalhadores para qualificação do SUS.

O objetivo deste artigo é refletir sobre as reformas efetuadas na Secretaria de Saúde do estado de São Paulo ante as propostas teóricas de gestão. Para apoiar o desenvolvimento desta reflexão, realizou-se uma breve pesquisa bibliográfica, nas bases de dados Lilacs e Scielo, nas quais se utilizaram as palavras-chave "Gestor", "Gestão", "SUS”, "Equidade” e "Integralidade". Selecionaram-se 22 fontes bibliográficas pertencentes ao período de 1993 a 2007.

\section{Resultados}

\section{A reforma da SES de São Paulo}

Os grandes avanços no desenvolvimento do Sistema Único de Saúde no estado de São Paulo, que incluem a extensão de acesso da população aos serviços de saúde, tanto na atenção primária como na atenção de maior complexidade, ocasionaram a percepção de novos problemas organizacionais e gerenciais, que exigem reflexão e estratégias de enfrentamento.

No atual estágio do SUS no estado de São Paulo, cabe à SES a responsabilidade geral de formulação e coordenação da política de saúde no estado, gerir e regular a assistência médica de maior complexidade, de caráter estadual ou regional, e prestar serviços de saúde pela rede de serviços estaduais que permanecem sob sua gerência. Além disso, a SES deve desenvolver ações de coordenação, supervisão, capacitação, acompanhamento e avaliação das ações de saúde de todos os municípios, auxiliando os sistemas municipais de saúde, quando for o caso ${ }^{2}$.

A Secretaria de Estado da Saúde é composta pelas seguintes coordenadorias: Coordenadoria de Regiões de Saúde (CRS), identificadora das necessidades dos serviços de saúde; Coordenadoria de Serviços de Saúde (CSS), operadora dos serviços de saúde; Coordenadoria de Gestão de Contratos de Serviços de Saúde (CGCSS), controladora da gestão dos serviços de saúde; Coordenadoria de Controle de Doenças (CCD), promotora da saúde coletiva; Coordenadoria de Ciência, Tecnologia e Insumos Estratégicos em Saúde (CCTIES), orientadora de incorporação e distribuição de novas tecnologias e insumos estratégicos de saúde; Coordenadoria de Planejamento de Saúde (CPS), gestora da informação e avaliadora do sistema; Coordenadoria Geral da Administração (CGA), administradora dos recursos materiais e financeiros; e Coordenadoria de Recursos Humanos (CRH), gestora dos recursos humanos.

Para se adaptar às novas exigências do SUS, a SES passou por uma importante reorganização, visando corrigir um conjunto de disfunções que foram se acumulando ao longo do tempo. No campo da assistência, substituiu uma estrutura em que as duas grandes coordenadorias (Interior e Região Metropolitana da Grande São Paulo) reproduziam as mesmas funções, tanto de regulação, avaliação e controle, compra de serviços e coordenação do sistema regional, como de prestação direta de serviços, de forma complementar aos municípios, através de suas regionais. Ao mesmo tempo, uma terceira coordenadoria, criada mais recentemente (CCSS), fazia a gestão dos serviços gerenciados pelas organizações sociais. Esta situação, na qual duas coordenadorias desenvolviam as mesmas funções e atividades, diferenciadas apenas pela estruturação regional, gerava dentro da Secretaria necessidade contínua de coordenação e adaptação dobrada às normas e políticas centrais, além de confundir em um mesmo espaço a função de diagnóstico de necessidade, compra e prestação de serviços, monitoramento e avaliação, com uma terceira coordenadoria atravessando as duas anteriores. Daí, portanto, o movimento de separar as funções de identificação de necessidades, regulação, 
planejamento e avaliação regional, assumidas pela nova Coordenadoria de Regiões de Saúde, daquelas de prestação dos serviços próprios ou em parceria, assumidas pela Coordenadoria de Serviços de Saúde².

A mudança da Coordenadoria de Saúde do Interior para Coordenadoria de Regiões de Saúde estimulou a instituição da nova configuração das Direções Regionais de Saúde através do Decreto $n^{\circ} 51.433$, de 28 de dezembro de 2006, que instituiu a mudança das denominações das Direções Regionais de Saúde (DIRs) para Departamentos Regionais de Saúde (DRS) e a passagem de 24 DIRs para 17 DRS. Os Departamentos Regionais de Saúde da Coordenadoria de Regiões de Saúde têm por finalidades contribuir para a qualidade de vida da população das respectivas regiões, coordenando, articulando, organizando e gerenciando o sistema de saúde locorregional; identificar a necessidade de compra de serviços de saúde; promover a articulação dos sistemas metropolitanos de saúde; avaliar, acompanhar e estabelecer a cooperação técnica dos sistemas de saúde; e tornar disponíveis e dar publicidade às informações de saúde e gerenciais que viabilizem o controle social do desempenho do sistema de saúde ${ }^{3}$.

A função da CPS passa a ser orientada para a gestão de informações, avaliação do sistema, identificação de necessidades e de boas práticas gerenciais em apoio à ação finalística das demais coordenadorias. A Coordenadoria dos Institutos de Pesquisa articulava ações de Vigilância Sanitária e Epidemiológica, porém regionalmente as equipes estavam administrativamente ligadas às DIRs, gerando conflito de comando. Ela concentrava todos os institutos de pesquisa com diferentes perfis. A reforma unifica o comando das ações de saúde coletiva na nova CCD e estrutura a Coordenadoria de Ciência, Tecnologia e Insumos Estratégicos, responsável por orientar a incorporação e o desenvolvimento de tecnologia, bem como gerir a compra e distribuição de insumos estratégicos, principalmente fármacos, imuno e hemoderivados.

A Coordenadoria de Recursos Humanos fortalece a capacidade de coordenar e orientar as ações de gestão de pessoal e as ações de desenvolvimento, pessoal e institucional. No campo do desenvolvimento de pessoal, incorpora a residência médica, coordena a implantação dos polos de educação permanente. No campo do desenvolvimento institucional, cria um Departamento de Apoio ao Desenvolvimento Institucional, constitui Observatório de Recursos Humanos, articula a política de humanização da SES, cria um serviço de Promoção da Qualidade de Vida no Trabalho. No campo de gestão de pessoal aprofunda-se a informatização da gestão de pessoal, instituem-se novos mecanismos de gestão flexível de pessoal e institui-se uma mesa de negociação coletiva.

A CGA fortalece sua capacidade de gestão administrativo-financeira, incorporando a Gestão do Fundes e desenvolvendo sua estrutura de compras e acompanhamento de contratos e convênios.

A CRS identifica a necessidade e contrata suplementarmente serviços não próprios do Sistema Único de Saúde (SUS/SP); orienta a contratualização dos serviços de saúde próprios e das organizações sociais de saúde; articula os sistemas metropolitanos de saúde; gerencia os sistemas de regulação locorregional; avalia, acompanha e estabelece a cooperação técnica dos sistemas de saúde municipais. A CSS atua no gerenciamento dos serviços próprios, estabelecendo a gestão do conhecimento e da informação.

A CGCSS fornece instrumentos para a contratação de serviços de saúde e realiza o controle administrativo e financeiro dos contratos e convênios de serviços de saúde. A CCD tem a função de coordenar os Sistemas de Vigilância Epidemiológica e Sanitária do Estado de São Paulo; realizar o armazenamento e a distribuição de imunobiológicos e diagnóstico laboratorial; organizar a documentação histórica e promover o ensino e a pesquisa.

A CCTIES coordena a política de aquisição de insumos estratégicos, o Sistema de Sangue Componentes e Derivados e a agenda de pesquisas de inovação e desenvolvimento tecnológico em saúde. A CPS tem a função de captar e consolidar dados para o delineamento do perfil de saúde da população do estado e conhecer a capacidade instalada em cada região; consolidar os processos de planejamento e avaliação dos serviços de saúde, seus resultados e impactos; desenvolver e transferir tecnologia e orientar os municípios nos processos de planejamento e gerenciamento da prestação de serviços de saúde e coordenar, controlar e avaliar o Sistema Estadual de Transplantes.

As atribuições da CGA são: promover a administração geral com acompanhamento, monitoramento e análise da execução orçamentária e financeira da SES; supervisionar o processo de elaboração da proposta parcial para o orçamento plurianual de investimento, da Lei de Diretrizes Orçamentárias e do Orçamento programa-anual; controlar e orientar as contratações de servi- 
ços especializados de saúde e as aquisições de medicamentos, equipamentos e outros produtos de saúde, destinadas ao atendimento de demandas extraordinárias. A CRH propõe e implementa políticas de desenvolvimento e formação de recursos humanos; organiza a formação de recursos humanos para o SUS, viabilizando a participação das instituições de ensino nos serviços de saúde do SUS; produz indicadores de recursos humanos do SUS e realiza pesquisas sobre o perfil da força de trabalho do setor saúde; realiza o planejamento e o controle dos recursos humanos da SES e promove a gestão de pessoal da SES.

Nesse sentido, a nova reforma foi orientada para racionalizar as ações da Secretaria, organizando-as em suas funções mais diretas: prestação direta de atenção à saúde; coordenação e compra de serviços para sistemas regionais de saúde; ações de saúde coletiva e vigilância à saúde; incorporação de tecnologia, desenvolvimento e distribuição de insumos estratégicos em saúde; planejamento, avaliação e gestão da informação; gestão administrativa financeira da SES; gestão e desenvolvimento de recursos humanos ${ }^{2}$.

As principais tendências no estado de São Paulo no que concerne à administração pública gerencial são representadas pela separação entre funções políticas e administrativas, distinção entre atividades exclusivas de Estado (formulação de políticas, arrecadação e regulação) das que podem ser realizadas por outros agentes, ênfase do controle dos procedimentos para controle dos produtos, atribuição clara de resultados (contratualismo), definição de formas de prestação de contas, governabilidade e governança.

\section{As pautas mais frequentes dos artigos de gestão}

A análise dos textos nos mostrou que a conquista da universalidade, da integralidade e da equidade não depende apenas da descentralização e da municipalização, mas da integração de esforços que aperfeiçoem e racionalizem os recursos existentes, construindo uma rede regionalizada e hierarquizada de serviços que atenda às questões de saúde relevantes de um ponto de vista epidemiológico em cada região. O fortalecimento do papel do gestor é importante para visualizar a continuidade do desenvolvimento do SUS e para a viabilização integral de suas premissas $^{4}$. Os indicadores de saúde são instrumentos de responsabilização da gestão de saúde pública e indicam a eficiência dos elementos de prestação setorial e peculiar de contas ${ }^{5}$.
É muito importante valorizar a participação social nos comitês ressaltando a necessidade de mobilizar e repolitizar o debate sobre o direito à saúde, na perspectiva de ampliação da participação e do poder de intervenção dos grupos sociais. A participação e o controle social são ações que devem ser desenvolvidas através do apoio aos Conselhos de Saúde, conferências e movimentos sociais que atuam na saúde ${ }^{1,6}$.

O planejamento é a ferramenta de gestão que possibilita aos gestores locais a reflexão sobre seus estilos, práticas e experiências visando a um planejamento de forma ascendente, participativo, comunicativo e estratégico. O Pacto pela Saúde em sua dimensão pacto pela gestão tem como diretriz o planejamento do SUS que pressupõe que cada esfera de gestão realize o seu planejamento, articulando-se de forma a fortalecer e consolidar os objetivos e diretrizes do SUS, contemplando as peculiaridades, necessidades e realidades de saúde locorregionais ${ }^{1,7}$.

A planificação, a programação e a avaliação dos sistemas e serviços de saúde visam instrumentalizar adequadamente a intervenção na situação de saúde. Dentre as responsabilidades gerais da gestão do SUS está o desenvolvimento, a partir da identificação das necessidades, de um processo de planejamento, regulação, programação pactuada e integrada da atenção à saúde, monitoramento e avaliação $0^{1,8}$.

Os indicadores de desigualdades em saúde devem ser selecionados a fim de definir prioridades de políticas públicas no Brasil. O SUS pode promover equidade no sentido de justiça sem comprometer o seu caráter universal e igualitário através da redução das desigualdades sociais. Dentre os objetivos da regionalização está o de garantir o direito à saúde, reduzir desigualdades sociais e territoriais e promover a equidade, ampliando a visão nacional dos problemas, em associação com a capacidade de diagnóstico e decisão locorregional que possibilite os meios adequados para a redução das desigualdades no acesso às ações e aos serviços de saúde existentes no país ${ }^{1,9,10}$.

$\mathrm{O}$ ato de avaliar só agrega valor quando o conhecimento e o uso das informações produzidas geram aprimoramento institucional e profissional. A educação permanente é considerada uma estratégia do SUS para formação e desenvolvimento de trabalhadores para o setor, a fim de promover a integralidade da atenção à saúde ${ }^{1,11}$.

A regulação da relação público/privado é uma necessidade para os avanços na gestão do sistema. A regulação tem como objetivo a produção de todas as ações diretas e finais da atenção à 
saúde, dirigida aos prestadores de serviços de saúde, públicos e privados. As ações da regulação da atenção à saúde compreendem a contratação, a regulação do acesso à assistência ou regulação assistencial, o controle assistencial, a avaliação da atenção à saúde, a auditoria assistencial e as regulamentações da vigilância epidemiológica e sanitária ${ }^{1,12}$.

A descentralização da saúde apresenta muitos desafios para os gestores da vigilância sanitária. Buscando aprofundar o processo de descentralização, o Ministério da Saúde propõe políticas, participação no cofinanciamento, cooperação técnica, avaliação, regulação, controle e fiscalização, descentralização dos processos administrativos relativos à gestão para as comissões intergestores bipartite ${ }^{1,13}$.

A gestão descentralizada do SUS é uma condição necessária para a efetiva melhora das condições e equidade em saúde. A regionalização é uma diretriz do Sistema Único de Saúde e um eixo estruturante do Pacto de Gestão e deve orientar a descentralização das ações e dos serviços de saúde e os processos de negociação e pactuação entre os gestores ${ }^{1,14}$. A gestão em saúde, mesmo sendo uma área com grande necessidade de proûssionalização, tanto pela seriedade de sua atividade-ûm como pela nobreza de sua função na sociedade, sofre com a falta de estudos e com os relatos diários da falta da gestão eûcaz. A educação em saúde tem como responsabilidades a formulação e a promoção da gestão da educação permanente na saúde e processos relativos a ela, orientados pela integralidade da atenção à saúde. A educação permanente deve ser considerada parte essencial de uma política de formação e desenvolvimento dos trabalhadores para a qualificação do SUS ${ }^{1,15}$.

A integralidade na atenção à saúde da população é o fruto da interação democrática dos sujeitos implicados na construção de respostas governamentais capazes de contemplar as diferenças expressas nas demandas em saúde. O Pacto pela Gestão refere que para que haja o fortalecimento da participação social é necessário apoiar os conselhos de saúde, as conferências de saúde e os movimentos sociais que atuam no campo da saúde, estimular a participação e a avaliação dos cidadãos nos serviços de saúde, apoiar a implantação e implementação de ouvidorias municipais e estatais ${ }^{1,16}$.

A equidade, na maioria dos países, é a oportunidade da utilização de serviços de saúde para necessidades iguais ${ }^{17}$. No contexto da otimização da efetividade e da equidade, a qualidade da aten- ção significa quanto as necessidades de saúde, existentes ou potenciais, estão sendo atendidas de forma otimizada pelos serviços de saúde, dado o conhecimento atual a respeito da distribuição, do reconhecimento, diagnóstico e manejo dos problemas e preocupações referentes à saúde ${ }^{18}$.

Avanços positivos na equidade ocorrem quando o processo de descentralização da política de saúde está presente, apresentando maior oferta $\mathrm{e}$ utilização de serviços em saúde no Brasil, conforme expõe a diretriz de descentralização do Pacto de Gestão $0^{1,19}$.

A formulação de políticas para a gestão de trabalho está diretamente articulada aos recursos humanos. Para que haja essa formulação, é necessário o entendimento de que o processo social envolve em sua construção questões relativas à gestão. No Pacto de Gestão, a diretriz de gestão do trabalho refere-se à política de recursos humanos para o SUS como eixo estruturante ${ }^{1,20}$.

Os Conselhos Gestores de Saúde devem promover a democracia participativa instituindo a participação em saúde, conforme é citado na diretriz da participação e do controle social do pacto de gestão. Pode-se dizer que o fato de um município ser pequeno não é necessariamente um obstáculo à inovação ou democratização da gestão ${ }^{1,21,22}$.

\section{Conclusão}

Identificou-se que a descentralização do SUS é um dos caminhos necessários a ser percorrido pelos gestores para a equidade e integralidade dos diversos âmbitos da saúde. A integralidade na atenção da saúde da população é o resultado da interação democrática dos sujeitos envolvidos nos processos de produção de saúde - e no atual estágio do SUS no estado de São Paulo, com a reforma da SES-SP, orientada para a racionalidade das ações desenvolvidas através da definição dos papéis nos diferentes níveis de gestão, gerando o reordenamento do trabalho, exigindo inovações e adaptações gerenciais significativas no âmbito das ações de saúde do estado. Desta forma, ocorrerá maior interação, pois cada coordenadoria tem novas funções, novas formas de olhar, novas estruturas, requerendo um aprendizado específico e novas formas e regras de trabalho nos processos de gestão, ante um sistema cada vez mais complexo.

Diante das mudanças organizacionais ocorridas na SES-SP, pode-se dizer que houve o fortalecimento do papel do gestor evidenciado a partir da extinção das DIRs, substituídas pelas DRS, 
que proporcionaram maior agilidade na interação do Secretário de Saúde com os municípios.

A capacitação e educação em saúde dos sujeitos envolvidos nos processos de gestão é também um dos caminhos para que ocorram a integralidade e a equidade na atenção à saúde, e esta é uma das ações em que a SES-SP deverá melhorar o desenvolvimento e a adequação ante as novas competências e práticas profissionais a fim de gerar a capacidade institucional necessária capaz de sustentar o funcionamento dessa nova organização.

O planejamento estratégico e a avaliação dos sistemas e serviços de saúde permitem a instrumentalização e a intervenção adequada de acordo com o diagnóstico situacional em saúde. A SES-SP no campo da assistência substituiu as coordenadorias (Interior e Região Metropolitana da Grande São Paulo) respectivamente pela Coordenadoria de Regiões de Saúde e Coordenadoria de Serviços de Saúde, propiciando planejamento e avaliação de forma mais estratégica.

A redefinição e o direcionamento das diversas funções de gestão da SES possibilitaram a racionalização dos recursos existentes; regulação de sistemas regionais de saúde; capacidade para identificação de necessidades de intervenção a partir da análise precisa dos indicadores de saúde; planejamento; avaliação e controle em âmbito regional; identificação e disseminação de novas tecnologias; precisão na prestação de apoio técnico e financeiro aos municípios, provendo a continuidade dos processos de descentralização com o intuito de que a cobertura das ações de saúde seja ampliada, diminuindo as desigualdades de atenção e promovendo a equidade nos processos de saúde através de uma gestão articulada entre o estado e os municípios.

O desenvolvimento desta pesquisa permitiunos a conclusão de que com os grandes avanços no desenvolvimento do SUS no estado de São Paulo, que incluem a extensão de acesso aos serviços de saúde à população, tanto na atenção primária como na atenção de maior complexidade, perceberam-se novos problemas organizacionais e gerenciais que exigem reflexão e estratégias de enfrentamento, estabelecendo como um dos principais desafios da política de saúde no estado a garantia da equidade e da integralidade nas ações do SUS, beneficiando as regiões de forma descentralizada e parcelas da população que ainda não conseguem ter pleno acesso às ações de saúde com eficácia e eficiência.

\section{Colaboradores}

RC Ermel trabalhou na concepção, redação e revisão final do artigo; FM Bigio, AFR Evangelista e MFP Gomes, na concepção, revisão da literatura e redação do artigo; LA Fracolli, na concepção, redação e revisão final do artigo. 


\section{Referências}

1. Brasil. Ministério da Saúde. Diretrizes Operacionais dos Pactos pela Vida, em Defesa do SUS e de Gestão. Brasília: Ministério da Saúde; 2006.

2. Seixas PHD. A reestruturação da SES-SP e as novas demandas para qualificação da gestão. In: São Paulo. Secretaria de Estado da Saúde. Planejamento de saúde: conhecimento \& ações 2006. São Paulo: Secretaria de Estado da Saúde; 2006. p. 271-283.

3. São Paulo. Decreto no 51.433 , de 28 de dezembro de 2006. Cria unidade na Coordenadoria de Regiões de Saúde, da Secretaria de Saúde, altera a denominação e dispõe sobre a reorganização das Direções Regionais de Saúde e dá providências correlatas. Diário Oficial do Estado de São Paulo 2006; 28 dez.

4. Bloch RA, Balassiano M. A democratização da gestão pública: as relações entre gestor, inovação e porte demográfico do município. Rev Adm Pública 2000; 34(1):145-164.

5. Campos SLF. A responsabilidade na gestão da saúde pública [dissertação]. São Paulo: Faculdade de Saúde Pública, Universidade de São Paulo; 2006.

6. Costa AM, Lionço T. Democracia e gestão participativa: uma estratégia para a eqüidade em saúde? Saúde Soc 2006; 5(2):47-55.

7. Cubas MR. Planejamento local: a fala do gerente de Unidade Básica de Saúde. Rev Bras Enferm 2005; 58(3):278-283.

8. Dobashi BF. Instrumentalizar adequadamente a intervenção na situação de saúde: um dos (muitos) dilemas do gestor municipal de saúde. Divulgação Saúde Debate 2004; 30:26-29.

9. Drachler ML, Côrtes SMV, Castro JD, Leite JCC. Propostas de metodologia para selecionar indicadores de desigualdades em saúde visando definir prioridades de políticas públicas no Brasil. Cien Saude Colet 2003; 8(2):461-470.

10. Paim JS. Eqüidade e reforma em sistemas de serviços de saúde: o caso do SUS. Saúde Soc 2006; 15(2):34-46.

11. Felisberto E. Da teoria à formulação de uma Política Nacional de Avaliação em Saúde: reabrindo o debate. Cien Saude Colet 2006; 11(3):553-563.

12. Ibanhes LC, Heimann LS, Junqueira V, Boaretto RC, Pessoto UC, Catarino U, Cortizo CT, Castro IEN, Rocha JL, Kayano J, Luiz OC, Barboza R, Telesi Júnior E. Governança e regulação na saúde: desafios para a gestão na região metropolitana de São Paulo. Cad Saude Publica 2007; 23(3):575-584.
13. Lima LD, Baptista TWF. Política e gestão da saúde no Brasil. In: De Seta MH, Pepe VLE, Oliveira GO. Gestão e vigilância sanitária: modos atuais do pensar e fazer. Rio de Janeiro: Editora Fiocruz; 2006. p. 89110.

14. Lucchese PTR. Eqüidade na gestão descentralizada do SUS: desafios para a redução de desigualdades em saúde. Cien Saude Colet 2003; 8(2):439-448.

15. Pessini L, Pereira LL, Chanes M. Gestão em saúde: assistência integral, valorização da pessoa, investimentos crescentes. Mundo Saúde 2006; 30(2):197-202.

16. Pinheiro R, Ferla A, Silva Júnior AG. A integralidade na atenção à saúde da população. Cien Saude Colet 2007; 12(2):343-349.

17. Van Doorslaer E, Wagstaff A, Rutten E. Equity in the finance and delivery of care: an international perspective. Oxford: Oxford University Press; 1993.

18. Starfield B. Atenção primária: equilíbrio entre necessidades de saúde, serviços e tecnologia. 2a ed. Brasília: Unesco/Ministério da Saúde; 2004.

19. Viana ALA, Fausto MCR, Lima LD. Política de saúde e eqüidade. São Paulo Perspec 2003; 17(1):58-68.

20. Vieira M. A gestão do trabalho no Sistema Único de Saúde. In: Escola Politécnica de Saúde Joaquim Venâncio, organizadora. Politicas de saúde: textos de apoio. Rio de Janeiro: Fiocruz; 2005. p. 99-116.

21. Wendhausen A, Cardoso SM. Processo decisório e Conselhos Gestores de Saúde: aproximações teóricas. Ver Bras Enferm 2007; 60(5):579-584.

22. Barata LRB, Tanaka OU, Mendes JDV. Por um processo de descentralização que consolide os princípios do Sistema Único de Saúde. Epidemiologia e Serviços de Saúde 2004; 13(1):15-24.

Artigo apresentado em 24/11/2008

Aprovado em 16/03/2009 